\title{
Current pumping performance of linear-type magnetic flux pump with use of feedback control circuit system
}

\section{$\operatorname{AUTHOR}(S):$}

Chung, Y; Hoshino, T; Nakamura, T

\section{CITATION:}

Chung, Y ... [et al]. Current pumping performance of linear-type magnetic flux pump with use of feedback control circuit system. IEEE TRANSACTIONS ON APPLIED SUPERCONDUCTIVITY 2006, 16(2): 1638-1641

\section{ISSUE DATE:}

2006-06

URL:

http://hdl.handle.net/2433/50546

\section{RIGHT:}

(c)2006 IEEE. Personal use of this material is permitted. However, permission to reprint/republish this material for advertising or promotional purposes or for creating new collective works for resale or redistribution to servers or lists, or to reuse any copyrighted component of this work in other works must be obtained from the IEEE. 


\title{
Current Pumping Performance of Linear-Type Magnetic Flux Pump With Use of Feedback Control Circuit System
}

\author{
Yoondo Chung, Tsutomu Hoshino, and Taketsune Nakamura
}

\begin{abstract}
It has already been investigated that current pumping of the linear-type magnetic flux pump (LTMFP) is controlled by frequency of 3-phase inverter. In this paper, based on these operating characteristics, a current compensator, which is composed of a LTMFP and a feedback control circuit system, has been introduced to compensate for the persistent current decay of the superconducting magnet. From the experiment, the current compensator has been demonstrated with the toroidal assembly of six $\mathrm{Nb}$-Ti magnets $543 \mathrm{mH}$ under the operating load current at $10 \mathrm{~A}$.
\end{abstract}

Index Terms-Current compensator, feedback control circuit system, linear-type magnetic flux pump.

\section{INTRODUCTION}

I N ORDER TO reduce the cooling cost of low temperature superconducting (LTS) magnets, broad studies using high temperature superconducting (HTS) magnets are advanced in superconducting applications. However, compared with the LTS, the HTS has a low $n$ value that is an intrinsic characteristic of the HTS, the low $n$ value is directly associated with the superconducting loop current decay [1]. Available HTS magnets have an intrinsically low $n$ value and thus cannot keep the persistent current in the closed superconducting loop. From the intrinsic characteristic of HTS, the technologies of current compensator to keep superconducting persistent current are expected to be very promising research in the HTS magnet applications [2], [3].

To use the HTS magnet in persistent current mode, there are two ways to overcome the decay of the current. One is development of high $n$ value superconductor. Another way is to introduce current compensator that does not affect current stability and regulation. We selected the later approach because we already presented a linear-type magnetic flux pump (LTMFP) to achieve the superconducting power supply [4].

As the LTMFP is based on the principle of the linear induction motor, the traveling field is generated by means of the 3-phase inverter and DC bias current. The operating characteristics of

Manuscript received September 19, 2005. This work was supported by Grant-in-Aid for exploratory research (no. 14655107) of JSPS and the 21st Century COE Program (no. 14213201) in Japan.

Y. Chung and T. Nakamura are with the Department of Electrical Engineering, Graduate School of Engineering, Kyoto University, Kyoto 615-8510, Japan (e-mail: yoondo@ asl.kuee.kyoto-u.ac.jp; tk_naka@kuee.kyoto-u.ac.jp).

T. Hoshino is with the Department of Electrical Engineering and Electronics, Faculty of Physical Science and Engineering, Meisei University, Tokyo 1918506, Japan (e-mail: thoshino@ee.meisei-u.ac.jp).

Digital Object Identifier 10.1109/TASC.2006.870452
LTMFP have already been investigated under various conditions. Moreover, it has been observed that the current pumping of the LTMFP can be controlled by frequency of the 3-phase inverter [5], [6].

The current pumping of the already developed flux pumps was controlled by the thermal-type superconducting persistent current switch (PCS) performances [7], [8]. In this paper, as a new approach, a new type current compensator, which consists of the LTMFP and a feedback control circuit system, is introduced. As the new current compensator directly controls the 3-phase inverter using the feedback control circuit system, the compensating current can be controlled without the PCS performances.

Since the feedback control circuit system [8] is installed at the outside of the cryostat, compared with the performances of the superconducting PCS, the operation is easily executed experimentally.

In this experiment, the proposed current compensator is periodically executed to inject $1 \mathrm{~mA}$ for persistent current decay under operating the load current at $10 \mathrm{~A}$. Especially, the LTMFP is performed with a toroidal-shape assembly of six Nb-Ti magnets which have $543 \mathrm{mH}$ inductance value. As the six $\mathrm{Nb}-\mathrm{Ti}$ magnets have five joints, there exist small contact resistances in such jointed points. The current decay in the closed superconducting current loop is caused. The contact resistance simulated low $n$ value of the HTS magnet. In order to evaluate the current pumping control using the introduced current compensator, the six Nb-Ti magnets are utilized as a superconducting magnet of the persistent current decay.

\section{Operation SEQuence of FeEdBack CONTROL CirCUIT System}

The feedback control circuit system is based on a bang-bang control technique [8]. The bang-bang unit which is one component of the feedback control circuit is main operating unit in order to control current pumping.

Fig. 1 provides the block diagram for control circuit by use of the operation of the feedback control circuit system. The control sequences are as follows:

First, the Hall voltage signal that corresponds to the superconducting loop current through the magnet applies to the voltage follower buffer as shown in the Fig. 2. The signal is amplified to adjust analog level for comparison with reference voltage level.

Second, the output signal of the comparator is clamped to logical level with a resistor and diodes, and feeds to local circuit. DQ flip-flop generates open or close signal of feedback 


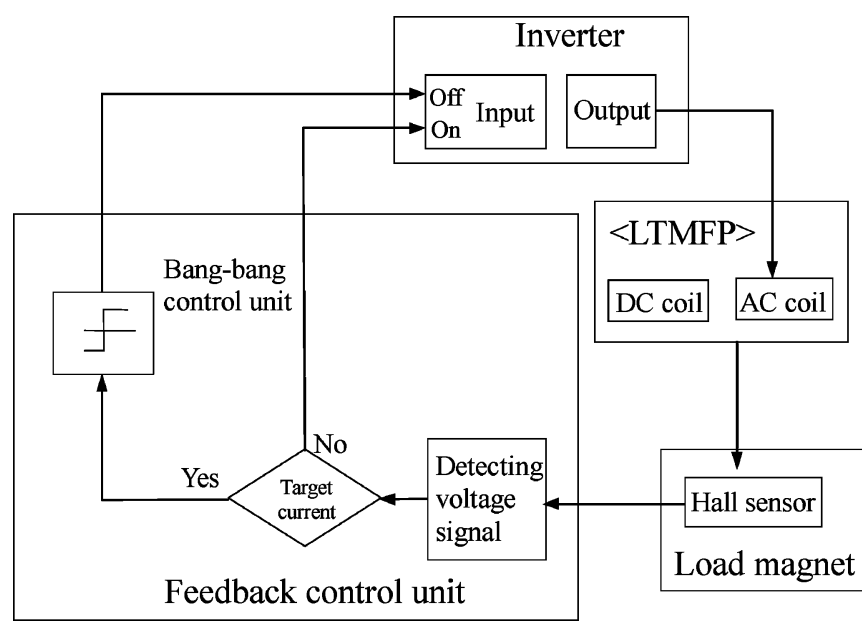

Fig. 1. Block diagram of the LTMFP system and feedback control circuit system.

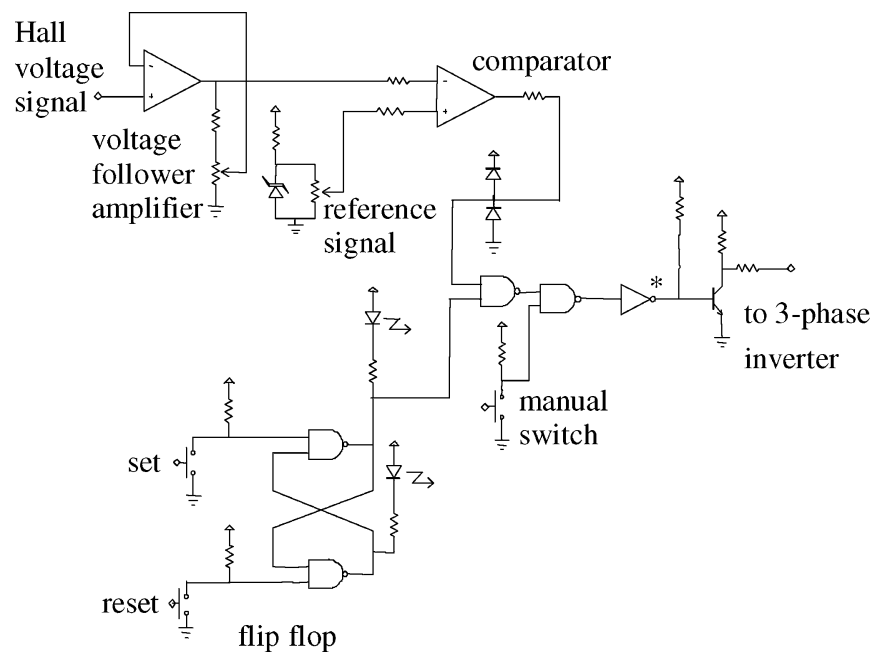

Fig. 2. Circuit diagram for feedback control circuit system.

circuit by manual operation. The signal from the comparator immediately changes the input signal operation. However, there is some delay time to turn-on and turn-off in the inverter operation, the delay time acts as adequate hysteresis function for stable bang-bang control.

Third, if the current value of detected signal is lower than the reference current, the 3-phase inverter turns on the input of the inverter. This is the operating state of the current compensator. However, if the level of detected signal exceeds the reference current, the control system turns off the input of the inverter. This is the persistent current mode.

The feedback control circuit system is designed to operate with the feedback sequence or the manual control manner. The over scale saturation of the operational amplifier for the comparator acts as a Schmidt trigger avoiding the chattering.

From the control sequences, the compensating operation for persistent current decay is performed as shown in Fig. 3. The operating load current $I_{\mathrm{op}}$ is compensated as little as the persistent current decay $\Delta I_{\mathrm{n}}$ by the periodical operation of the feedback control circuit system. Especially, due to the superconducting

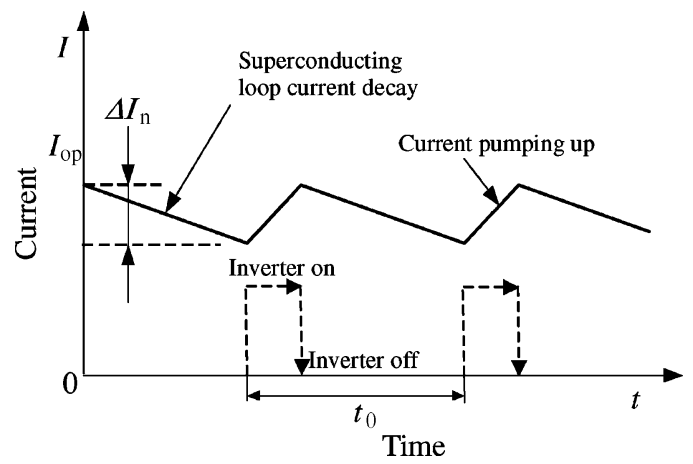

Fig. 3. Schematic waveform of superconducting loop current and compensating operation by the inverter.

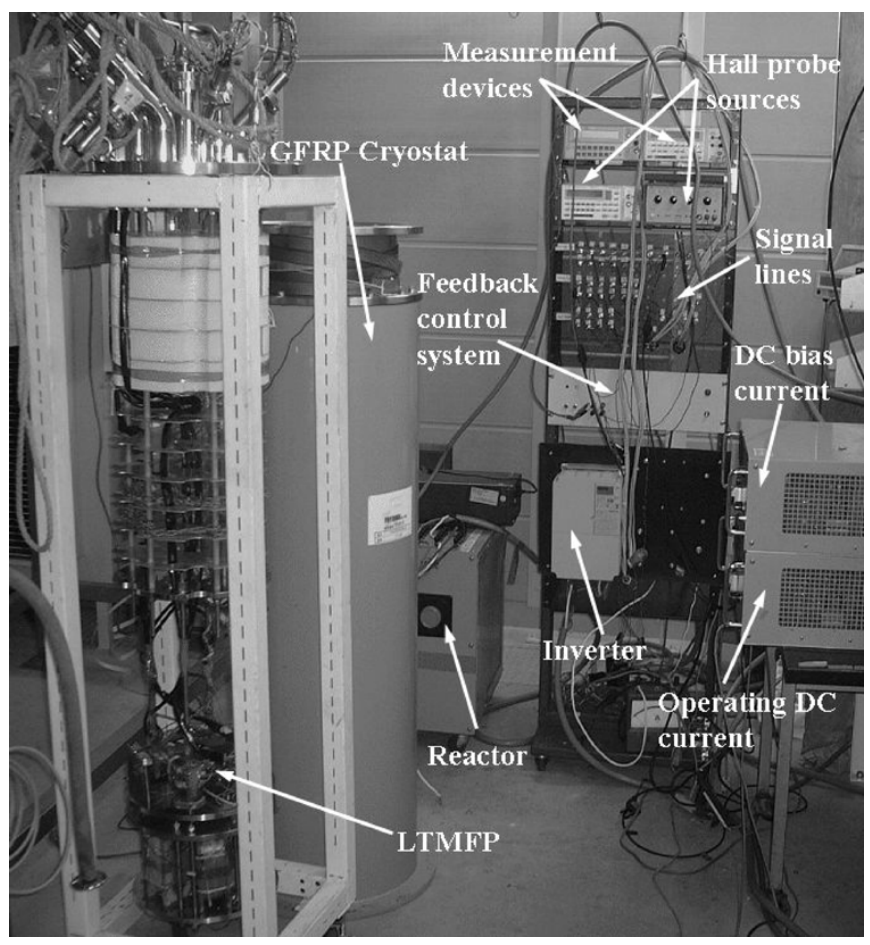

Fig. 4. Photograph of the assembled compensator control system with LTMFP system.

loop current decay of the closed load magnet, the current compensator is periodically operated by the feedback control circuit system.

\section{EXPERIMENTAL SETUP AND PROCEDURE}

Cryogenic part of the LTMFP with load magnet and other experimental setup parts are shown in Fig. 4. The experimental system of the current compensator system mainly consists of four parts; the LTMFP system, the power sources, the feedback control circuit system, and measuring system. The cryogenic part that hung at the rack is put in the GFRP cryostat.

Fig. 5 shows the connection diagram of the current compensator system and sensors. The two Hall sensors and a couple of voltage taps are installed in the system. The two Hall sensors measure the temporal variation of the magnetic flux density at the central air gap and the current pumping in the load magnet, respectively. The voltage taps of the feedback control system 


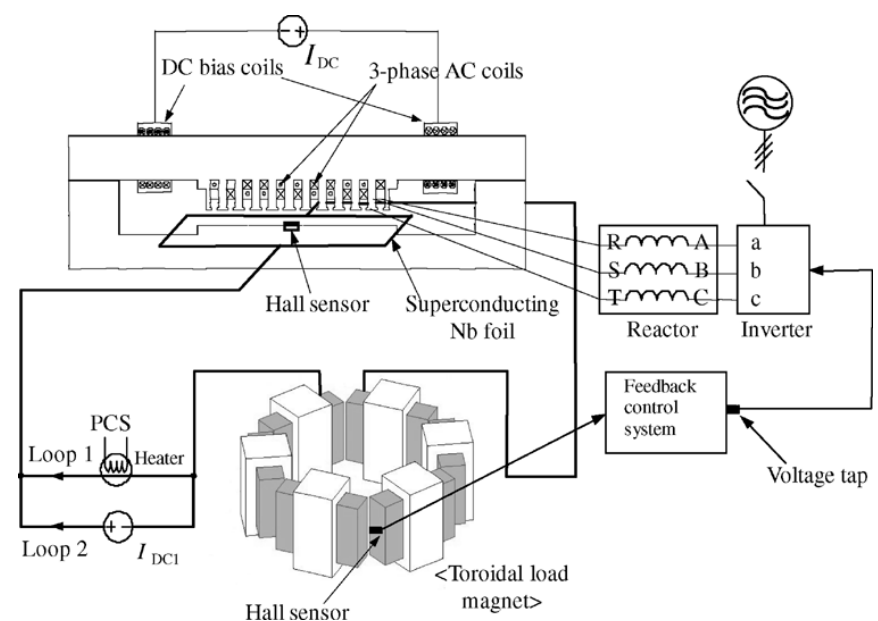

Fig. 5. Connection diagram of LTMFP system, feedback control system and sensors.

measure the inverter on/off voltage. The six $\mathrm{Nb}$-Ti magnets, a sheet of Nb foil, and a PCS are connected to keep superconducting current loop.

The experimental sequences of the current compensator are explained as follows:

Step 1) the DC current is applied to the DC bias coil, but the coil stays in superconducting state. In addition, the PCS turns off by the heater power.

Step 2) the external DC current source $I_{\mathrm{DC} 1}$ supplies the load current into the load magnet and LTMFP (Loop 2 in Fig. 5).

Step 3) the heating current of the PCS is slowly turned off, to turn on the PCS, and the external operation DC current of $10 \mathrm{~A}$ is turned off at the same time. Now, the current through Loop 1 flows in a persistent current (so called persistent current mode).

Step 4) due to the superconducting loop current decay of the load magnet, the current compensator is periodically turned on by the feedback control circuit system. The current compensator system compensates little by little the target current value.

Step 5) after compensating for target current value, the current compensator is turned off by the feedback control system; it maintains the superconducting persistent current mode.

\section{EXPERIMENTAL RESULTS}

In this experiment, the current compensator was periodically carried out in order to generate as little as $1 \mathrm{~mA}$ with the operating load current at $10 \mathrm{~A}$. In addition, before the compensating current test, the decay of the superconducting loop current was investigated.

As the six $\mathrm{Nb}-\mathrm{Ti}$ magnets system were connected by five joints, there exist small contact resistances in such jointed points. These resistances cause the current decay in the closed superconducting current loop.

Fig. 6 shows the measured result of the decay of the persistent current loop (Loop 1); the initial current is $10.367 \mathrm{~A}$ and the decayed current becomes 10.355 A during 90 minutes. After all,

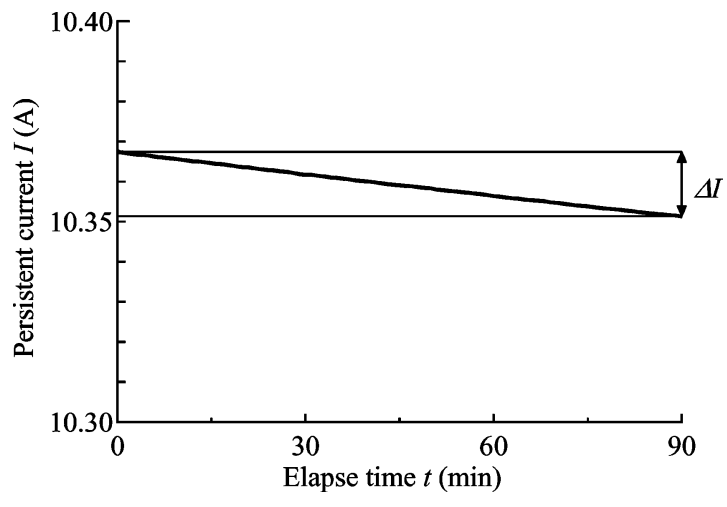

Fig. 6. Measured result of the decay of the persistent current loop.

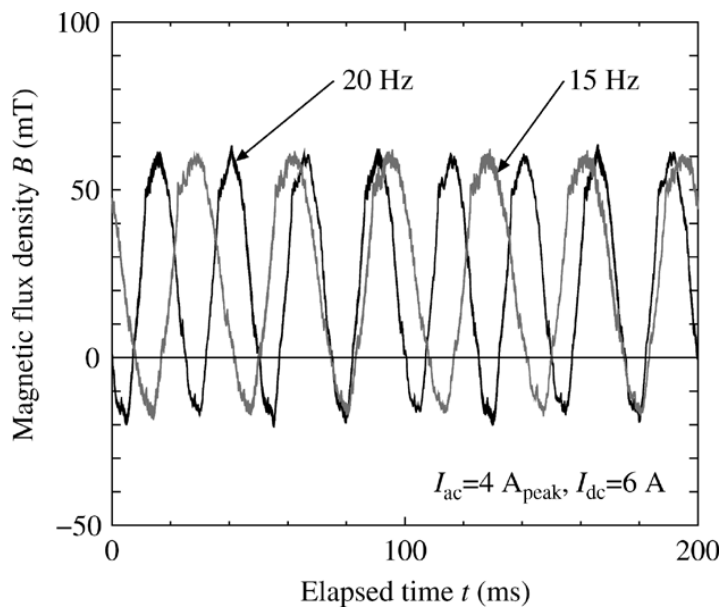

Fig. 7. Measured results of the temporal variation of the magnetic flux density at the central air gap with DC bias current at $6 \mathrm{~A}$ and $\mathrm{AC}$ current at $4 \mathrm{~A}_{\text {peak }}$.

the decay of the persistent loop current of $12 \mathrm{~mA}$ was measured for 90 minutes. The decay time constant was calculated as 1295 $\mathrm{h}$, and equivalent joint resistance of the superconducting current loop was obtained as $0.116 \mu \Omega$.

Fig. 7 shows the measured results of temporal variation of the magnetic flux density at the central air gap with DC bias current at $6 \mathrm{~A}$ and $\mathrm{AC}$ current at $4 \mathrm{~A}_{\text {peak }}$ of 15 and $20 \mathrm{~Hz}$, respectively. With these driving conditions, the positive and negative peak values are $60 \mathrm{mT}$ and $-20 \mathrm{mT}$, respectively. As the traveling magnetic field by the 3-phase inverter generates pumped current in the $\mathrm{Nb}$ foil which is installed in the air gap, the current compensator controls the inverter in order to generate the pumped current as little as $1 \mathrm{~mA}$.

Fig. 8 shows the measured results of the periodical operation with DC bias current at $6 \mathrm{~A}$ and $\mathrm{AC}$ current at $4 \mathrm{~A}_{\text {peak }}$ $(f=15 \mathrm{~Hz})$ using the feedback control circuit. It has been investigated that the introduced current compensator produces the compensating current $1.5 \mathrm{~mA}$ with operating load current at 10 A. During the operation, the peak current of the inverter $I_{0}$ is 4.10 A. The compensating time of the current pumping $1.5 \mathrm{~mA}$ is 28.3 seconds. Due to the soft start function of the inverter, the build-up rate of the inverter output is $0.85 \mathrm{~A} / \mathrm{s}$, and build-down rate is $-1.07 \mathrm{~A} / \mathrm{s}$.

Due to the superconducting loop current decay of the toroidal assembly of six $\mathrm{Nb}-\mathrm{Ti}$ magnets, the closed superconducting 


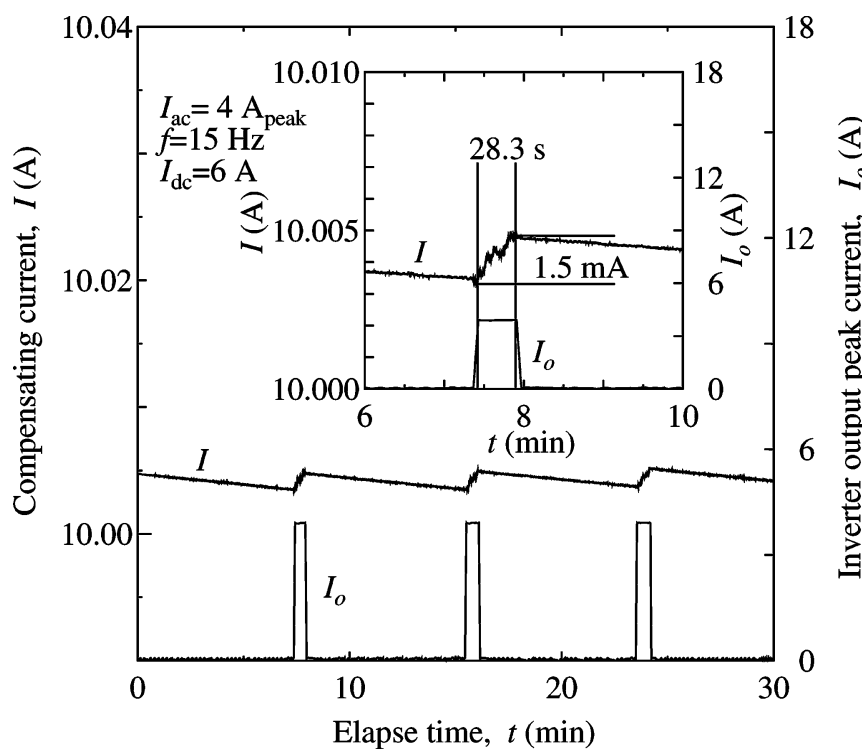

Fig. 8. Measured results of compensating current and inverter on/off voltage by the feedback control circuit system with DC bias current at $6 \mathrm{~A}$ and $\mathrm{AC}$ current at $4 \mathrm{~A}_{\text {peak }}(f=15 \mathrm{~Hz})$.

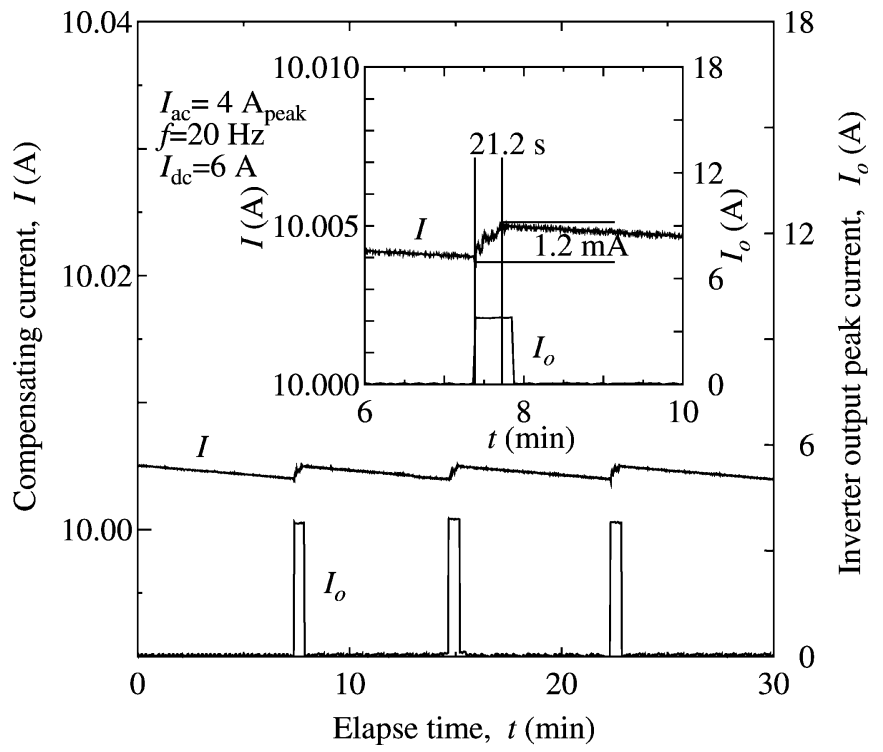

Fig. 9. Measured results of compensating current and inverter on/off voltage by the feedback control circuit system with DC bias current at $6 \mathrm{~A}$ and $\mathrm{AC}$ current at $4 \mathrm{~A}_{\text {peak }}(f=20 \mathrm{~Hz})$.

loop of the LTMFP system caused the persistent current decay as fast as $1 \mathrm{~mA}$ per 7.5 minutes as shown in Fig. 6.

Fig. 9 shows the periodically measured results for the compensating current $1.2 \mathrm{~mA}$ with $\mathrm{DC}$ bias current at $6 \mathrm{~A}$ and $\mathrm{AC}$ current at $4 \mathrm{~A}_{\text {peak }}(f=20 \mathrm{~Hz})$. By the experimental results, the peak current of the inverter output $I_{0}$ is $4.13 \mathrm{~A}$. In addition, the operating time of the compensating current $1.2 \mathrm{~mA}$ is 21.2 seconds.

\section{CONCLUSION AND DisCUSSION}

In this experiment, the introduced current compensator has been evaluated using a feedback control circuit system and LTMFP system. Furthermore, the feedback control circuit system presents one solution for the superconducting loop current decay without PCS performances. Finally, from these performances, the compensating current about $1 \mathrm{~mA}$ has been executed using the current compensator under the load magnet $543 \mathrm{mH}$.

The intervals between the pumping operation and the on-off operation of the inverter are caused by the operating delay of the inverter. This problem can be solved by the use of the AC current source instead of the inverter.

In addition, in order to operate the LTMFP under the higher operating current, it has been expected that the plural superconducting $\mathrm{Nb}$ foils (parallel connections) is required.

Based on the operating characteristics of the introduced current compensator, a new control method for compensated current was demonstrated under the under small scale superconducting magnet.

\section{REFERENCES}

[1] Y. Iwasa, "HTS magnets: stability; protection; cryogenics; economics; current stability/protection activities at FBML," Cryogenics, vol. 43, pp. 303-316, Mar. 2003.

[2] — "Microampere flux pump for superconducting NMR magnets Part1; basic concept and microtesla flux measurement," Cryogenics, vol. 41, pp. 385-391, May 2001.

[3] S. K. Jeong, "Experiment of flux pump for high temperature superconductor insert coils of NMR magnets," J. Korea Inst. Appl. Supercond. Cryogenics, vol. 3, no. 2, pp. 15-20, Nov. 2001.

[4] I. Muta, H. Tsukiji, T. Hoshino, and E. Mukai, "Electrical characteristics of fully superconducting synchronous generator in persistent excitation mode," IEEE Trans. Magn., vol. 28, no. 1, pp. 434-437, Jan. 1992.

[5] Y. Chung, I. Muta, T. Hoshino, and T. Nakamura, "Performances of a linear type magnetic flux pump for compensating a little decremented persistent current of HTS magnets," IEEE Trans. Appl. Supercond., vol. 14, no. 2, pp. 1723-1726, Jun. 2004

[6] Y. Chung, I. Muta, T. Hoshino, T. Nakamura, and M. H. Sohn, "Design and performance of compensator for decremental persistent current in HTS magnets using linear type magnetic flux pump," Cryogenics, vol. 44, no. 11, pp. 839-844, Nov. 2004.

[7] Y. S. Yoon, S. J. Lee, H. M. Kim, and T. K. Ko, "Analysis of the operational characteristics of a heater-trigger type high- $T_{\mathrm{C}}$ superconducting power supply," IEEE Trans. Appl. Supercond., vol. 9, no. 4, pp. 4708-4714, Dec. 1999.

[8] S. K. Jeong, S. H. In, and S. H. Kim, "Superconducting micro flux pump using a cryotron-like switch," IEEE Trans. Appl. Supercond., vol. 13, no. 2, pp. 668-671, Jun. 2003.

[9] J. F. Gieras and Z. J. Piech, Linear Synchronous Motors Transportation and Automation Systems. : CRC Press, 2000, pp. 157-171. 